\title{
Global Dynamics of a Predator-Prey Model with Stage Structure and Delayed Predator Response
}

\author{
Lili Wang and Rui Xu \\ Institute of Applied Mathematics, Shijiazhuang Mechanical Engineering College, No. 97 Heping West Road, \\ Shijiazhuang, Hebei 050003, China
}

Correspondence should be addressed to Rui Xu; rxu88@163.com

Received 21 May 2013; Accepted 27 October 2013

Academic Editor: Qi-Ru Wang

Copyright (C) 2013 L. Wang and R. Xu. This is an open access article distributed under the Creative Commons Attribution License, which permits unrestricted use, distribution, and reproduction in any medium, provided the original work is properly cited.

A Holling type II predator-prey model with time delay and stage structure for the predator is investigated. By analyzing the corresponding characteristic equations, the local stability of each of feasible equilibria of the system is discussed. The existence of Hopf bifurcations at the coexistence equilibrium is established. By means of the persistence theory on infinite dimensional systems, it is proven that the system is permanent if the coexistence equilibrium exists. By using Lyapunov functionals and LaSalle's invariance principle, it is shown that the predator-extinction equilibrium is globally asymptotically stable when the coexistence equilibrium is not feasible, and the sufficient conditions are obtained for the global stability of the coexistence equilibrium.

\section{Introduction}

In population dynamics, the functional response of predator to prey density refers to the change in the density of prey attacked per unit time per predator as the prey density changes [1]. Based on experiments, Holling [2] suggested three different kinds of functional responses for different kinds of species to model the phenomena of predation, which made the standard Lotka-Volterra system more realistic. The most popular functional response used in the modelling of predator-prey systems is Holling type II with $\varphi(x)=f x /(1+$ $m x)$ which takes into account the time a predator uses in handing the prey being captured. There has been a large body of work about predator-prey systems with Holling type II functional responses, and many good results have been obtained (see, e.g., $[1,3,4]$ ).

Time delays of one type or another have been incorporated into biological models by many researchers. We refer to the monographs of Gopalsamy [5], Kuang [6], and Wangersky and Cunningham [7] on delayed predator-prey systems. In these research works, it is shown that a time delay could cause a stable equilibrium to become unstable and cause the population to fluctuate. Hence, delay differential equations exhibit more complex dynamics than ordinary differential equations. Time delay due to gestation is a common example, since generally the consumption of prey by the predator throughout its past history decides the present birth rate of the predator. In [7], Wangersky and Cunningham proposed and studied the following non-Kolmogorov-type predatorprey model:

$$
\begin{gathered}
\dot{x}(t)=x(t)\left(r_{1}-a x(t)-a_{1} y(t)\right), \\
\dot{y}(t)=a_{2} x(t-\tau) y(t-\tau)-r_{2} y(t) .
\end{gathered}
$$

In this model, it is assumed that a duration of $\tau$ time units elapses when an individual prey is killed and the moment when the corresponding addition is made to the predator population.

In natural world, there are many species whose individuals have a history that can be divided into two stages immature and mature. Usually the dynamics-eating habits of predator are often quite different in different stages. Generally speaking, the immature predators are raised by their parents and do not have the ability to attack prey, so the rate at which they attack prey and the reproductive rate can be ignored. Hence, it is of ecological importance to investigate predator-prey models with stage structure. In recent years, the predator-prey population models with stage structure have received much attraction (see, e.g., [8-10]). In [10], it was assumed that feeding on prey can only make contribution 
to the increasing of the physique of the predator and does not make contribution to the reproductive ability, and the following strengthen type predator-prey model with stage structure was studied:

$$
\begin{gathered}
\dot{x}(t)=x(t)\left(r-a x(t)-\frac{a_{1} y_{2}(t)}{1+m x(t)}\right), \\
\dot{y}_{1}(t)=e y_{2}(t)-\left(r_{1}+D\right) y_{1}(t), \\
\dot{y}_{2}(t)=D y_{1}(t)-r_{2} y_{2}(t)+\frac{a_{2} x(t) y_{2}(t)}{1+m x(t)},
\end{gathered}
$$

where $x(t)$ represents the density of the prey at time $t$ and $y_{1}(t)$ and $y_{2}(t)$ represent the densities of the immature and the mature predator at time $t$, respectively. $a_{1} x /(1+m x)$ describes the Holling type II functional response; $a_{1}$ and $m$ represent the effects of capturing rate and handling time, respectively. $a$ denotes the intraspecific competition rate of the prey, $e$ denotes the birth rate of immature predator, $r$ denotes the intrinsic growth rate of the prey, $a_{2} / a_{1}$ denotes the rate of conversing prey into new mature predator, $r_{1}$ denotes the death rate of the immature predator, $r_{2}$ denotes the death rate of the mature predator, and $D$ denotes the rate of immature predator becoming mature predator. All parameters are positive constants. In [10], sufficient conditions were derived in for the global asymptotic stability of nonnegative equilibria of the model by constructing suitable Lyapunov functions.

Motivated by the work of Wangersky and Cunningham [7] and Tian and $\mathrm{Xu}$ [10], we are concerned with the combined effects of the stage structure for the predator and time delay due to the gestation of mature predator on the global dynamics of a predator-prey model with Holling type II functional response. To this end, we consider the following delay differential system:

$$
\begin{gathered}
\dot{x}(t)=x(t)\left(r-a x(t)-\frac{a_{1} y_{2}(t)}{1+m x(t)}\right), \\
\dot{y}_{1}(t)=e y_{2}(t)-\left(r_{1}+D\right) y_{1}(t), \\
\dot{y}_{2}(t)=D y_{1}(t)-r_{2} y_{2}(t)+\frac{a_{2} x(t-\tau) y_{2}(t-\tau)}{1+m x(t-\tau)},
\end{gathered}
$$

where the meanings of the variables $x, y_{1}, y_{2}$, and the parameters $a, r, e, a_{1}, a_{2}, r_{1}, r_{2}, D$, are the same as those of system (2) and the constant $\tau \geq 0$ denotes the time delay due to the gestation of the mature predator. This is based on the assumption that the change rate of predators depends on the number of prey and of mature predators present at some previous time.

The initial conditions for system (3) take the form

$$
\begin{array}{ccc}
x(\theta)=\phi(\theta), & y_{1}(\theta)=\psi_{1}(\theta), & y_{2}(\theta)=\psi_{2}(\theta), \\
\theta \in[-\tau, 0), & \phi(0)>0, \quad \psi_{1}(0)>0, \quad \psi_{2}(0)>0,
\end{array}
$$

where

$$
\begin{array}{r}
\left(\phi(\theta), \psi_{1}(\theta), \psi_{2}(\theta)\right) \in C\left([-\tau, 0], R_{+0}^{3}\right), \\
R_{+0}^{3}=\left\{\left(x_{1}, x_{2}, x_{3}\right): x_{1} \geq 0, x_{2} \geq 0, x_{3} \geq 0\right\} .
\end{array}
$$

By the fundamental theory of functional differential equations [11], it is well known that system (3) has a unique solution $\left(x(t), y_{1}(t), y_{2}(t)\right)$ satisfying initial conditions (4). Further, it is easy to show that all solutions of system (3) are defined on $[0,+\infty)$ and remain positive for all $t \geq 0$.

The paper is organized as follows. In the next section, by analyzing the corresponding characteristic equations, the local stability of each of nonnegative equilibria of system (3) is discussed and the existence of Hopf bifurcations at the coexistence equilibrium is established. In Section 3, permanence of the system (3) is proved by means of the persistence theory on infinite dimensional systems. In Section 4, by using Lyapunov functionals and LaSalle's invariance principle, sufficient conditions are received for the global asymptotic stability of the predator-extinction equilibrium and the coexistence equilibrium.

\section{Local Stability and Hopf Bifurcation}

In this section, we discuss the local stability of each of feasible equilibria of system (3) and the existence of Hopf bifurcations at the coexistence equilibrium.

It is easy to show that system (3) always has a trivial equilibrium $E_{0}(0,0,0)$ and a predator-extinction equilibrium $E_{1}(r / a, 0,0)$. Furthermore, if the following holds.

(H1) $a_{2} r\left(r_{1}+D\right)>(a+m r)\left[r_{2}\left(r_{1}+D\right)-e D\right]>0$, then system (3) has a coexistence equilibrium $E^{*}=\left(x^{*}, y_{1}^{*}, y_{2}^{*}\right)$, where

$$
\begin{gathered}
x^{*}=\frac{r_{2}\left(r_{1}+D\right)-e D}{\left(a_{2}-m r_{2}\right)\left(r_{1}+D\right)+m e D}, \quad y_{1}^{*}=\frac{e}{r_{1}+D} y_{2}^{*}, \\
y_{2}^{*}=\frac{a_{2}\left(r_{1}+D\right)\left(r-a x^{*}\right) x^{*}}{a_{1}\left[r_{2}\left(r_{1}+D\right)-e D\right]} .
\end{gathered}
$$

We now study the local stability of the trivial equilibrium $E_{0}(0,0,0)$ and the predator-extinction equilibrium $E_{1}(r / a, 0,0)$.

The characteristic equation of system (3) at $E_{0}(0,0,0)$ is

$$
(\lambda-r)\left[\lambda^{2}+\left(r_{1}+r_{2}+D\right) \lambda+r_{2}\left(r_{1}+D\right)-e D\right]=0 .
$$

Hence, $E_{0}(0,0,0)$ is always unstable since ( 7$)$ has a positive $\operatorname{root} \lambda=r$.

The characteristic equation of system (3) at $E_{1}(r / a, 0,0)$ is

$$
(\lambda+r)\left[P(\lambda)+Q(\lambda) e^{-\lambda \tau}\right]=0,
$$

where $P(\lambda)=\lambda^{2}+\left(r_{1}+r_{2}+D\right) \lambda+r_{2}\left(r_{1}+D\right)-e D, Q(\lambda)=$ $-a_{2} r \lambda /(a+m r)-a_{2} r\left(r_{1}+D\right) /(a+m r)$. 
Noting that (8) has a negative root $\lambda=-r$, the other roots of (8) are determined by the following:

$$
P(\lambda)+Q(\lambda) e^{-\lambda \tau}=0
$$

Denote

$$
f(\lambda)=P(\lambda)+Q(\lambda) e^{-\lambda \tau}
$$

If $a_{2} r\left(r_{1}+D\right)>(a+m r)\left[r_{2}\left(r_{1}+D\right)-e D\right]$, then

$$
\begin{gathered}
f(0)=-\frac{a_{2} r\left(r_{1}+D\right)-(a+m r)\left[r_{2}\left(r_{1}+D\right)-e D\right]}{a+m r}<0, \\
\lim _{\lambda \rightarrow+\infty} f(\lambda)=+\infty .
\end{gathered}
$$

Hence, $f(\lambda)=0$ has at least one positive root; the predatorextinction equilibrium $E_{1}(r / a, 0,0)$ is unstable.

If $a_{2} r\left(r_{1}+D\right)<(a+m r)\left[r_{2}\left(r_{1}+D\right)-e D\right]$, it is readily seen from (9) that $E_{1}$ is locally asymptotically stable when $\tau=0$. Denote

$$
F(y)=|P(i y)|^{2}-|Q(i y)|^{2}=y^{4}+h_{1} y^{2}+h_{2},
$$

where

$$
\begin{aligned}
h_{1} & =\left(r_{1}+r_{2}+D\right)^{2}-2\left[r_{2}\left(r_{1}+D\right)-e D\right]-\frac{a_{2}^{2} r^{2}}{(a+m r)^{2}} \\
> & \left(r_{1}+D\right)^{2}+r_{2}^{2}+2 e D-\frac{\left[r_{2}\left(r_{1}+D\right)-e D\right]^{2}}{\left(r_{1}+D\right)^{2}} \\
& =\frac{\left[\left(r_{1}+D\right)^{2}+e D\right]^{2}+2 e D\left[r_{2}\left(r_{1}+D\right)-e D\right]}{\left(r_{1}+D\right)^{2}}>0, \\
h_{2}= & \frac{(a+m r)^{2}\left[r_{2}\left(r_{1}+D\right)-e D\right]^{2}-a_{2}^{2} r^{2}\left(r_{1}+D\right)^{2}}{(a+m r)^{2}}>0 .
\end{aligned}
$$

Hence, $F(y)=0$ has no positive root. By Theorem 3.4.1 in [6], we know that if $a_{2} r\left(r_{1}+D\right)<(a+m r)\left[r_{2}\left(r_{1}+D\right)-e D\right]$, the predator-extinction equilibrium $E_{1}(r / a, 0,0)$ is locally asymptotically stable for all $\tau \geq 0$.

Concluding the above discussions, we obtain the following results.

Theorem 1. For system (3), one has the following.

(i) The trivial equilibrium $E_{0}(0,0,0)$ is always unstable.

(ii) If $a_{2} r\left(r_{1}+D\right)>(a+m r)\left[r_{2}\left(r_{1}+D\right)-e D\right]$, the predator-extinction equilibrium $E_{1}(r / a, 0,0)$ is unstable; if $a_{2} r\left(r_{1}+D\right)<(a+m r)\left[r_{2}\left(r_{1}+D\right)-e D\right], E_{1}$ is locally asymptotically stable for all $\tau \geq 0$.

In the following, we discuss the local stability of the coexistence equilibrium $E^{*}=\left(x^{*}, y_{1}^{*}, y_{2}^{*}\right)$ and the existence of Hopf bifurcations at $E^{*}$. $\left.y_{2}^{*}\right)$ is

The characteristic equation of system (3) at $E^{*}=\left(x^{*}, y_{1}^{*}\right.$,

$$
\lambda^{3}+p_{2} \lambda^{2}+p_{1} \lambda+p_{0}+\left(q_{2} \lambda^{2}+q_{1} \lambda+q_{0}\right) e^{-\lambda \tau}=0,
$$

where

$$
\begin{gathered}
p_{0}=\left(-r+2 a x^{*}+\frac{a_{1} y_{2}^{*}}{\left(1+m x^{*}\right)^{2}}\right)\left[r_{2}\left(r_{1}+D\right)-e D\right], \\
p_{1}=r_{2}\left(r_{1}+D\right)-e D \\
-\left(r-2 a x^{*}-\frac{a_{1} y_{2}^{*}}{\left(1+m x^{*}\right)^{2}}\right)\left(r_{1}+r_{2}+D\right), \\
p_{2}=r_{1}+r_{2}+D-r+2 a x^{*}+\frac{a_{1} y_{2}^{*}}{\left(1+m x^{*}\right)^{2}}, \\
q_{0}=\frac{a_{2} x^{*}}{1+m x^{*}}\left(r_{1}+D\right)\left(r-2 a x^{*}\right) \\
q_{1}=\frac{a_{2} x^{*}}{1+m x^{*}}\left(r-2 a x^{*}-r_{1}-D\right) \\
q_{2}=-\frac{a_{2} x^{*}}{1+m x^{*}} .
\end{gathered}
$$

When $\tau=0$, (14) becomes

$$
\lambda^{3}+\left(p_{2}+q_{2}\right) \lambda^{2}+\left(p_{1}+q_{1}\right) \lambda+p_{0}+q_{0}=0 .
$$

By calculations, we obtain that

$$
\begin{gathered}
p_{2}+q_{2}=r_{1}+D+2 a x^{*}-r+\frac{e D}{r_{1}+D}+\frac{a_{1} y_{2}^{*}}{\left(1+m x^{*}\right)^{2}} \\
p_{0}+q_{0}=\frac{a_{1} y_{2}^{*}}{\left(1+m x^{*}\right)^{2}}\left[r_{2}\left(r_{1}+D\right)-e D\right] .
\end{gathered}
$$

If (H1) holds, $p_{0}+q_{0}>0$. Hence, by the Routh-Hurwitz criterion, we know that the coexistence equilibrium $E^{*}=$ $\left(x^{*}, y_{1}^{*}, y_{2}^{*}\right)$ is locally asymptotically stable provided the following.

(H2) $p_{2}+q_{2}>0,\left(p_{2}+q_{2}\right)\left(p_{1}+q_{1}\right)-\left(p_{0}+q_{0}\right)>0$, and $E^{*}$ is unstable if one of the inequality in $(\mathrm{H} 2)$ is reversed.

Clearly, $\lambda=i \omega(\omega>0)$ is a root of (14) if and only if

$$
\begin{aligned}
& -i \omega^{3}-p_{2} \omega^{2}+i p_{1} \omega+p_{0} \\
& +\left(-q_{2} \omega^{2}+i q_{1} \omega+q_{0}\right)(\cos \omega \tau-i \sin \omega \tau)=0 .
\end{aligned}
$$

Separating real and imaginary parts, we have

$$
\begin{aligned}
-\omega^{3}+p_{1} \omega & =\left(q_{0}-q_{2} \omega^{2}\right) \sin \omega \tau-q_{1} \omega \cos \omega \tau, \\
-p_{2} \omega^{2}+p_{0} & =-q_{1} \omega \sin \omega \tau-\left(q_{0}-q_{2} \omega^{2}\right) \cos \omega \tau .
\end{aligned}
$$

Squaring and adding the two equations of (19), we obtain

$$
\omega^{6}+l_{2} \omega^{4}+l_{1} \omega^{2}+l_{0}=0
$$


where

$$
\begin{aligned}
l_{0}= & p_{0}^{2}-q_{0}^{2}=\left(p_{0}+q_{0}\right)\left(p_{0}-q_{0}\right) \\
= & -\frac{a_{1} y_{2}^{*}}{\left(1+m x^{*}\right)^{2}}\left[r_{2}\left(r_{1}+D\right)-e D\right]^{2} \\
& \times\left[2\left(r-2 a x^{*}\right)-\frac{a_{1} y_{2}^{*}}{\left(1+m x^{*}\right)^{2}}\right], \\
l_{1}= & p_{1}^{2}-2 p_{0} p_{2}+2 q_{0} q_{2}-q_{1}^{2} \\
= & \frac{a_{1} y_{2}^{*}}{\left(1+m x^{*}\right)^{2}}\left[\left(D+r_{1}\right)^{2}+r_{2}^{2}+2 e D\right] \\
& \times\left[\frac{a_{1} y_{2}^{*}}{\left(1+m x^{*}\right)^{2}}-2\left(r-2 a x^{*}\right)\right]+\left(r-2 a x^{*}\right)^{2} \\
& \times\left[\left(D+r_{1}\right)^{2}+2 e D+\frac{e D\left(2 r_{2}\left(r_{1}+D\right)-e D\right)}{\left(r_{1}+D\right)^{2}}\right], \\
l_{2}= & p_{2}^{2}-2 p_{1}-q_{2}^{2} \\
= & \left(D+r_{1}\right)^{2}+\left(r-2 a x^{*}-\frac{a_{1} y_{2}^{*}}{\left(1+m x^{*}\right)^{2}}\right)^{2} \\
& +\frac{e D}{\left(r_{1}+D\right)^{2}}\left[2\left(r_{1}+D\right)^{2}+2 r_{2}\left(r_{1}+D\right)-e D\right] .
\end{aligned}
$$

Letting $\omega^{2}=z$, (20) can be rewritten as

$$
z^{3}+l_{2} z^{2}+l_{1} z+l_{0}=0 .
$$

Denote

$$
l(z)=z^{3}+l_{2} z^{2}+l_{1} z+l_{0}
$$

If $\Delta=l_{2}^{2}-3 l_{1}>0$, then $l^{\prime}(z)=0$ has two unequal roots:

$$
z_{1}^{*}=\frac{-l_{2}+\sqrt{\Delta}}{3}, \quad z_{2}^{*}=\frac{-l_{2}-\sqrt{\Delta}}{3} .
$$

By Lemma 2.1 in [12], we can obtain the following conclusion.

Lemma 2. Suppose that (H1) is satisfied and $z_{1}^{*}$ is defined by (24).

(i) If $p_{0}<q_{0}$, then (22) has at least one positive root.

(ii) If $p_{0} \geq q_{0}$ and $\Delta<0$, then (22) has no positive roots.

(iii) If $p_{0} \geq q_{0}$, then (22) has positive roots if and only if $z_{1}^{*}>0, l\left(z_{1}^{*}\right) \leq 0$.

Without loss of generality, we assume that (22) has three positive roots, denoted by $z_{k}(k=1,2,3)$, and $\omega_{k}=\sqrt{z_{k}}(k=$ $1,2,3)$ are the three positive roots of (20) correspondingly.
Denote

$$
\begin{aligned}
& \tau_{k}^{(j)} \\
& =\frac{1}{\omega_{k}}\left[\operatorname { a r c s i n } \left(\left(q_{2} \omega_{k}^{5}+\left(p_{2} q_{1}-p_{1} q_{2}-q_{0}\right) \omega_{k}^{3}\right.\right.\right. \\
& \left.+\left(p_{1} q_{0}-p_{0} q_{1}\right) \omega_{k}\right) \\
& \left.\left.\times\left(q_{2}^{2} \omega_{k}^{4}-\left(2 q_{0} q_{2}-q_{1}^{2}\right) \omega_{k}^{2}+q_{0}^{2}\right)^{-1}\right)+2 j \pi\right], \\
& k=1,2,3 ; j=0,1,2, \ldots \text {; }
\end{aligned}
$$

then $\pm i \omega_{k}$ is a pair of purely imaginary roots of (14) with $\tau=$ $\tau_{k}^{(j)}, k=1,2,3 ; j=0,1,2, \ldots$.

Define $\tau_{0}=\tau_{k_{0}}^{(0)}=\min _{1 \leq k \leq 3}\left\{\tau_{k}^{(0)}\right\}, \omega_{0}=\omega_{k_{0}}$. By Lemma 2.2 in [12], the following result can be obtained.

Lemma 3. Suppose that (H1) and (H2) are satisfied.

(i) If $p_{0} \geq q_{0}$ and $\Delta<0$, then all roots of (14) have negative real parts for all $\tau \geq 0$.

(i) If $p_{0}<q_{0}$, or $p_{0} \geq q_{0}, z_{1}^{*}>0, l\left(z_{1}^{*}\right) \leq 0$, then all roots of (14) have negative real parts when $\tau \in\left[0, \tau_{0}\right)$.

Let $\lambda(\tau)=\alpha(\tau)+i \omega(\tau)$ be the root of (14) satisfying $\alpha\left(\tau_{0}\right)=0, \omega\left(\tau_{0}\right)=\omega_{0}$. The conditions in the following lemma ensure that the transversality condition holds.

Lemma 4. If $l^{\prime}\left(z_{0}\right) \neq 0$ and the conditions (ii) in Lemma 3 are satisfied, then

$$
\left.\frac{\mathrm{d} \operatorname{Re} \lambda(\tau)}{\mathrm{d} \tau}\right|_{\tau=\tau_{0}}>0
$$

Proof. Differentiating (14) with respect $\tau$, it follows that

$$
\begin{aligned}
& {\left[\left(3 \lambda^{2}+2 p_{2} \lambda+p_{1}\right)+\left(2 q_{2} \lambda+q_{1}\right) e^{-\lambda \tau}\right.} \\
& \left.-\left(q_{2} \lambda^{2}+q_{1} \lambda+q_{0}\right) \tau e^{-\lambda \tau}\right] \frac{\mathrm{d} \lambda}{\mathrm{d} \tau} \\
& \quad=\left(q_{2} \lambda^{2}+q_{1} \lambda+q_{0}\right) \lambda e^{-\lambda \tau}
\end{aligned}
$$

which yields

$$
\begin{aligned}
\left(\frac{\mathrm{d} \lambda(\tau)}{\mathrm{d} \tau}\right)^{-1}= & \frac{3 \lambda^{2}+2 p_{2} \lambda+p_{1}}{-\lambda\left(\lambda^{3}+p_{2} \lambda^{2}+p_{1} \lambda+p_{0}\right)} \\
& +\frac{2 q_{2} \lambda+q_{1}}{\lambda\left(q_{2} \lambda^{2}+q_{1} \lambda+q_{0}\right)}-\frac{\tau}{\lambda}
\end{aligned}
$$

On substituting $\tau=\tau_{0}$ into (28), then

$$
\left.\left(\frac{\mathrm{dRe} \lambda(\tau)}{\mathrm{d} \tau}\right)^{-1}\right|_{\tau=\tau_{0}}=\frac{l^{\prime}\left(z_{0}\right)}{q_{1}^{2} \omega_{0}^{2}+\left(q_{0}-q_{2} \omega_{0}^{2}\right)^{2}}
$$


Since $l^{\prime}\left(z_{0}\right) \neq 0$,

$$
\left.\frac{\mathrm{d} \operatorname{Re} \lambda(\tau)}{\mathrm{d} \tau}\right|_{\tau=\tau_{0}} \neq 0 .
$$

If $\mathrm{d} \operatorname{Re} \lambda(\tau) /\left.\mathrm{d} \tau\right|_{\tau=\tau_{0}}<0$, there exists a root $\lambda(\tau)=\alpha(\tau)+i \omega(\tau)$ satisfying $\alpha(\tau)>0$ for $\tau<\tau_{0}$ and close to $\tau_{0}$, which contradicts (ii) in Lemma 3. The proof is complete. results.

Applying Lemmas 3 and 4, we obtain the following

Theorem 5. For system (3), suppose that (H1) and (H2) are satisfied.

(i) If $p_{0} \geq q_{0}$ and $\Delta<0$, then the coexistence equilibrium $E^{*}=\left(x^{*}, y_{1}^{*}, y_{2}^{*}\right)$ is locally asymptotically stable for all $\tau \geq 0$.

(i) If $p_{0}<q_{0}$, or $p_{0} \geq q_{0}, z_{1}^{*}>0, l\left(z_{1}^{*}\right) \leq 0$, then $E^{*}$ is locally asymptotically stable for $0<\tau<\tau_{0}$ and is unstable for $\tau>\tau_{0}$.

(iii) If the conditions in (ii) are satisfied, and $l^{\prime}\left(z_{0}\right) \neq 0$, then system (3) exhibits the Hopf bifurcation at $E^{*}$ when $\tau=$ $\tau_{0}$.

Example 6. In (2), let $a=0.5, a_{1}=0.4, a_{2}=0.4, e=0.1$, $D=0.1, r=1, r_{1}=0.1, r_{2}=0.25$, and $m=0.5$. System (3) with above coefficients has a unique coexistence equilibrium $E^{*}(0.67,1.11,2.22)$. A direct calculation shows that $p_{2}+q_{2} \approx$ $0.42>0,\left(p_{2}+q_{2}\right)\left(p_{1}+q_{1}\right)-\left(p_{0}+q_{0}\right) \approx 0.04>0, p_{0}-$ $q_{0} \approx-0.01<0, \tau_{0} \approx 5.67$. By Theorem 5 , we see that the positive $E^{*}$ is locally asymptotically stable if $0<\tau<\tau_{0}$ and is unstable if $\tau>\tau_{0}$. Numerical simulation illustrates our result (see Figure 1).

\section{Permanence}

In the following, we show that system (3) is permanent.

Definition 7. System (3) is said to be permanent if there are positive constants $m_{1}, m_{2}, M_{1}$, and $M_{2}$ such that each positive solution $\left(x(t), y_{1}(t), y_{2}(t)\right)$ of system (3) satisfies

$$
\begin{gathered}
m_{1} \leq \liminf _{t \rightarrow+\infty} x(t) \leq \limsup _{t \rightarrow+\infty} x(t) \leq M_{1}, \\
m_{2} \leq \liminf _{t \rightarrow+\infty} y_{i}(t) \leq \limsup _{t \rightarrow+\infty} y_{i}(t) \leq M_{2}, \quad i=1,2 .
\end{gathered}
$$

Firstly, we prove that system (3) is ultimately bounded.

Lemma 8. If $r_{2}\left(r_{1}+D\right)-e D>0$, the arbitrary solution $(x(t)$, $\left.y_{1}(t), y_{2}(t)\right)$ of system (3) is ultimately bounded.

Proof. Let $\left(x(t), y_{1}(t), y_{2}(t)\right)$ be any positive solution of system (3). Define

$$
L(t)=a_{2} x(t-\tau)+k y_{1}(t)+a_{1} y_{2}(t),
$$

where $k$ is a constant satisfying $a_{1} D /\left(r_{1}+D\right)<k<a_{1} r_{2} / e$.
Calculating the derivative of $L(t)$ along positive solutions of system (3), we get

$$
\begin{aligned}
\dot{L}(t)= & a_{2} x(t-\tau)[r-a x(t-\tau)] \\
& -\left(r_{1}+D-\frac{a_{1} D}{k}\right) k y_{1}-\left(r_{2}-\frac{e k}{a_{1}}\right) a_{1} y_{2} \\
\leq & a_{2} x(t-\tau)[r+\delta-a x(t-\tau)] \\
& -\delta\left[a_{2} x(t-\tau)+k y_{1}+a_{1} y_{2}\right] \\
\leq & \frac{a_{2}(r+\delta)^{2}}{4 a}-\delta L(t),
\end{aligned}
$$

where $\delta=\min \left\{r_{1}+D-a_{1} D / k, r_{2}-e k / a_{1}\right\}$.

It is easy to know that $\delta>0$ for $a_{1} D /\left(r_{1}+D\right)<k<a_{1} r_{2} / e$. According to (33), we get

$$
\limsup _{t \rightarrow+\infty} L(t) \leq \frac{a_{2}(r+\delta)^{2}}{4 a \delta} .
$$

If we choose

$$
\begin{gathered}
M_{1}=\frac{(r+\delta)^{2}}{4 a \delta}, \quad M_{2}=\frac{a_{2}(r+\delta)^{2}}{4 a k \delta}, \\
M_{3}=\frac{a_{2}(r+\delta)^{2}}{4 a a_{1} \delta},
\end{gathered}
$$

then, for $\varepsilon>0$ sufficiently small, there exists a $T_{1}>0$ such that if $t>T_{1}$,

$$
x(t)<M_{1}+\varepsilon, \quad y_{1}(t)<M_{2}+\varepsilon, \quad y_{2}(t)<M_{3}+\varepsilon .
$$

That is, the arbitrary solution $\left(x(t), y_{1}(t), y_{2}(t)\right)$ of system (3) is ultimately bounded if $r_{2}\left(r_{1}+D\right)-e D>0$. This completes the proof.

Next, we use the persistence theory on infinite dimensional systems introduced by Hale and Waltman in [13] to prove the permanence of system (3).

Let $X$ be a complete metric space. Suppose that $X^{0} \subset X$, $X_{0} \subset X$, and $X^{0} \cap X_{0}=\phi$. Assume that $T(t)$ is a $C_{0}$ semigroup on $X$ satisfying

$$
T(t): X^{0} \longrightarrow X^{0}, \quad T(t): X_{0} \longrightarrow X_{0} .
$$

Let $T_{b}(t)=\left.T(t)\right|_{X_{0}}$ and $A_{b}$ be the global attractor for $T_{b}(t)$. The following is a small variant of Theorem $4.1 \mathrm{in}$ [13].

Lemma 9 (see [13]). Suppose that $T(t)$ satisfies (37) and one has the following.

(i) There is a $t_{0} \geq 0$ such that $T(t)$ is compact for $t>t_{0}$;

(ii) $T(t)$ is point dissipative in $X$;

(iii) $\widetilde{A}_{b}=\bigcup_{x \in A_{b}} \omega(x)$ is isolated and has an acyclic covering $\widetilde{M}$, where

$$
\widetilde{M}=\left\{M_{1}, M_{2}, \ldots, M_{n}\right\} ;
$$

(iv) $W^{s}\left(M_{i}\right) \cap X^{0}=\phi$ for $i=1,2, \ldots, n$. 

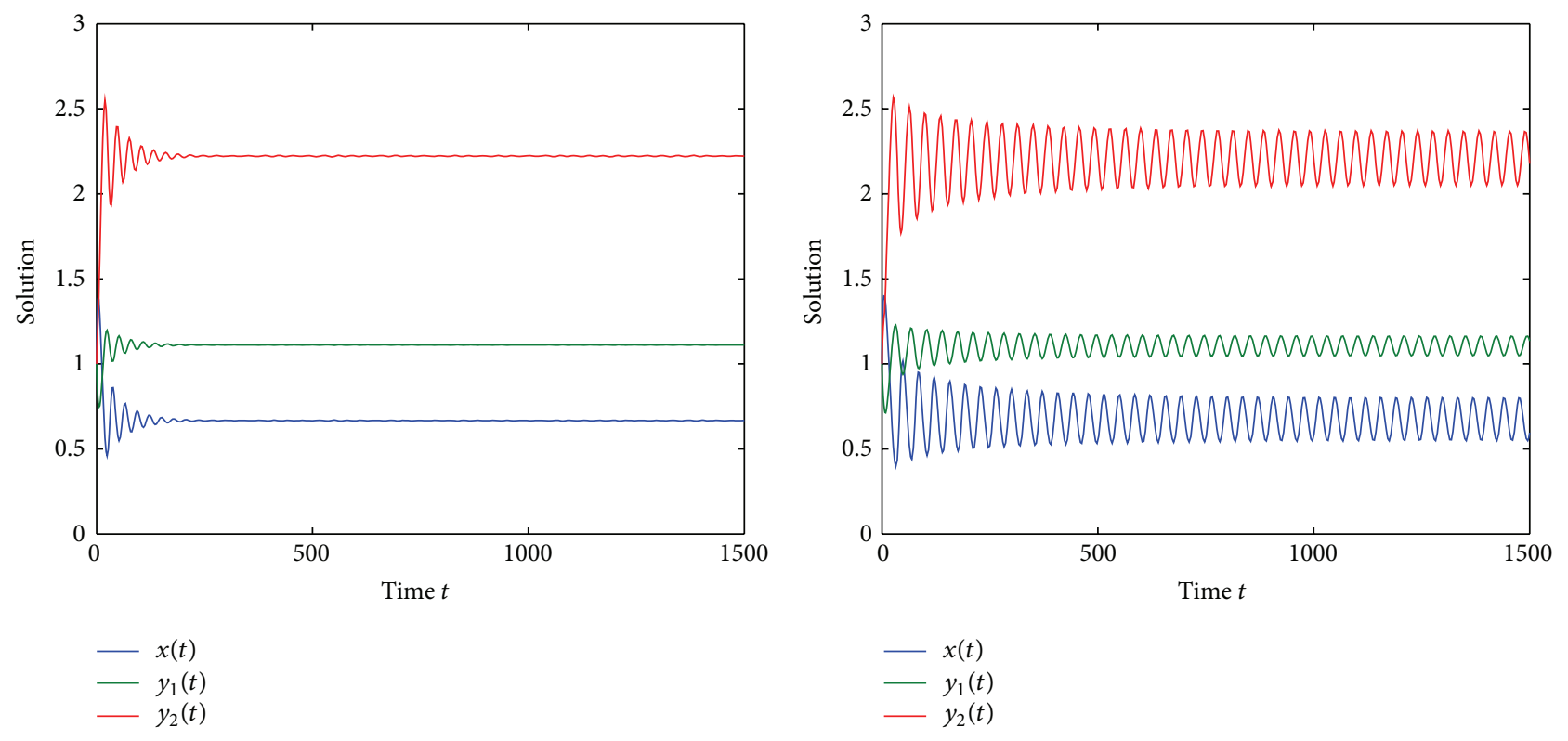

(a)

(b)

FIGURE 1: The numerical solution of system (3) with $a=0.5, a_{1}=0.4, a_{2}=0.4, e=0.1, D=0.1, r=1, r_{1}=0.1, r_{2}=0.25, m=0.5$; (a) $\tau=3$, (b) $\tau=6$ and $\left(\phi, \psi_{1}, \psi_{2}\right)=(1 ; 1 ; 1)$.

Then, $X_{0}$ is a uniform repeller with respect to $X^{0}$; that is, there is an $\varepsilon>0$ such that, for any $x \in X^{0}, \liminf _{t \rightarrow+\infty} d(T(t) x$, $\left.X_{0}\right) \geq \varepsilon$, where $d$ is the distance of $T(t) x$ from $X_{0}$.

Now we state and prove the permanence of system (3).

Theorem 10. If (H1) holds, then system (3) is permanent.

Proof. We need only to show that the boundaries of $R_{+0}^{3}$ repel positive solutions of system (3) uniformly.

Let $C^{+}\left([-\tau, 0], R_{+0}^{3}\right)$ denote the space of continuous functions mapping $[-\tau, 0]$ into $R_{+0}^{3}$. Define

$$
\begin{gathered}
C_{1}=\left\{\left(\phi, \psi_{1}, \psi_{2}\right) \in C^{+}\left([-\tau, 0], R_{+0}^{3}\right): \phi(\theta) \equiv 0, \theta \in[-\tau, 0]\right\} \\
C_{2}=\left\{\left(\phi, \psi_{1}, \psi_{2}\right) \in C^{+}\left([-\tau, 0], R_{+0}^{3}\right): \phi(\theta)>0\right. \\
\left.\psi_{1}(\theta) \equiv 0, \psi_{2}(\theta) \equiv 0, \theta \in[-\tau, 0]\right\}
\end{gathered}
$$

Denote $X=C^{+}\left([-\tau, 0], R_{+0}^{3}\right), C_{0}=C_{1} \cup C_{2}, C^{0}=\operatorname{int} C^{+}([-\tau$, $\left.0], R_{+0}^{3}\right)$.

Now, we verify that the conditions in Lemma 9 are satisfied. According to the definition of $C^{0}$ and $C_{0}$, it is easy to know that $C^{0}$ and $C_{0}$ are positively invariant, so condition (ii) in Lemma 9 is satisfied. The solution of system (3) is ultimately bounded if (H1) holds by Lemma 8 . Thus, by the smoothing property of solutions of delay differential equations introduced in [6, Theorem 2.2.8], condition (i) is satisfied.
Next, we verify condition (iii) in Lemma 9. There are two constant solutions in $C_{0}: \widehat{E}_{0} \in C_{1}, \widehat{E}_{1} \in C_{2}$ corresponding to $E_{0}(0,0,0)$ and $E_{1}(r / a, 0,0)$, respectively, which satisfy

$$
\begin{gathered}
\widehat{E}_{0}=\left\{\left(\phi, \psi_{1}, \psi_{2}\right) \in C^{+}\left([-\tau, 0], R_{+0}^{3}\right): \phi(\theta) \equiv 0,\right. \\
\left.\psi_{1}(\theta) \equiv 0, \psi_{2}(\theta) \equiv 0, \theta \in[-\tau, 0]\right\}, \\
\widehat{E}_{1}=\left\{\left(\phi, \psi_{1}, \psi_{2}\right) \in C^{+}\left([-\tau, 0], R_{+0}^{3}\right): \phi(\theta)=\frac{r}{a},\right. \\
\left.\psi_{1}(\theta) \equiv 0, \psi_{2}(\theta) \equiv 0, \theta \in[-\tau, 0]\right\} .
\end{gathered}
$$

If $\left(x(t), y_{1}(t), y_{2}(t)\right)$ is a solution of system (3) initiating from $C_{1}$, then

$$
\begin{gathered}
\dot{y}_{1}(t)=e y_{2}(t)-\left(r_{1}+D\right) y_{1}(t), \\
\dot{y}_{2}(t)=D y_{1}(t)-r_{2} y_{2}(t) .
\end{gathered}
$$

Obviously, if (H1) holds, $y_{1}(t) \rightarrow 0$ and $y_{2}(t) \rightarrow 0$ as $t \rightarrow$ $+\infty$. If $\left(x(t), y_{1}(t), y_{2}(t)\right)$ is a solution of system (3) initiating from $C_{2}$, then $\dot{x}(t)=x(t)[r-a x(t)]$, which yields $x(t) \rightarrow r / a$, as $t \rightarrow+\infty$. Noting that $C_{1} \cap C_{2}=\phi$, we know the invariant $\widehat{E}_{0}$ and $\widehat{E}_{1}$ are isolated. So, $\left\{\widehat{E}_{0}, \widehat{E}_{1}\right\}$ is isolated and is an acyclic covering satisfying conditions (iii) in Lemma 9.

We now show that $W^{s}\left(\widehat{E}_{0}\right) \cap C^{0}=\phi$ and $W^{s}\left(\widehat{E}_{1}\right) \cap C^{0}=\phi$.

Assume that $W^{s}\left(\widehat{E}_{0}\right) \cap C^{0} \neq \phi$. Then there is a positive solution $\left(x(t), y_{1}(t), y_{2}(t)\right)$ of system (3) satisfying

$$
\lim _{t \rightarrow+\infty} x(t)=0, \quad \lim _{t \rightarrow+\infty} y_{1}(t)=0, \quad \lim _{t \rightarrow+\infty} y_{2}(t)=0
$$


Then for $\varepsilon>0$ sufficiently small such that $r-a_{1} \varepsilon>0$, there exists a $T_{2}>0$ such that if $t>T_{2}$,

$$
-\varepsilon<y_{2}(t)<\varepsilon \text {. }
$$

Together with (43) and the first equation of system (3), we derive that

$$
\dot{x}(t)>x(t)\left[r-a x(t)-a_{1} \varepsilon\right] .
$$

Thus, $\liminf \operatorname{in}_{t \rightarrow+\infty} x(t) \geq\left(r-a_{1} \varepsilon\right) / a$, which contradicts $\lim _{t \rightarrow+\infty} x(t)=0$. Then $W^{s}\left(\widehat{E}_{0}\right) \cap C^{0}=\phi$.

Assume that $W^{s}\left(\widehat{E}_{1}\right) \cap C^{0} \neq \phi$. Then, there is a positive solution $\left(x(t), y_{1}(t), y_{2}(t)\right)$ of system (3) satisfying

$$
\lim _{t \rightarrow+\infty} x(t)=\frac{r}{a}, \quad \lim _{t \rightarrow+\infty} y_{1}(t)=0, \quad \lim _{t \rightarrow+\infty} y_{2}(t)=0 .
$$

Since (H1) holds, we can choose $\varepsilon>0$ small enough such that

$$
r_{2}\left(r_{1}+D\right)-e D<\frac{a_{2}(r / a-\varepsilon)\left(r_{1}+D\right)}{1+m(r / a-\varepsilon)} .
$$

Since $\lim _{t \rightarrow+\infty} x(t)=r / a$, for $\varepsilon>0$ satisfying (46), there exists a $T_{3}>0$ such that if $t>T_{3}$,

$$
\frac{r}{a}-\varepsilon<x(t)<\frac{r}{a}+\varepsilon
$$

From the last two equations of system (3), it is easy to know that if $t>T_{3}+\tau$,

$$
\begin{gathered}
\dot{y}_{1}(t)=e y_{2}(t)-\left(r_{1}+D\right) y_{1}(t), \\
\dot{y}_{2}(t) \geq D y_{1}(t)-r_{2} y_{2}(t)+\frac{a_{2}(r / a-\varepsilon) y_{2}(t-\tau)}{1+m(r / a-\varepsilon)} .
\end{gathered}
$$

Let us consider the following auxiliary system:

$$
\begin{gathered}
\dot{z}_{1}=e z_{2}(t)-\left(r_{1}+D\right) z_{1}(t), \\
\dot{z}_{2}=D z_{1}(t)-r_{2} z_{2}(t)+\frac{a_{2}(r / a-\varepsilon) z_{2}(t-\tau)}{1+m(r / a-\varepsilon)},
\end{gathered}
$$

with initial conditions (4).

Consider the following matrix $A_{\varepsilon}$ defined by

$$
A_{\varepsilon}=\left(\begin{array}{cc}
-\left(r_{1}+D\right) & e \\
D & -r_{2}+\frac{a_{2}(r / a-\varepsilon)}{1+m(r / a-\varepsilon)}
\end{array}\right) .
$$

Since $A_{\varepsilon}$ admits positive off-diagonal elements, the PerronFrobenius theorem implies that there is a positive eigenvector $v=\left(v_{1}, v_{2}\right)$ for the maximum root $\alpha$ of $A_{\varepsilon}$. Since (46) holds, it is shown that the maximum root $\alpha>0$ by a simple computation.

Let $z(t)=\left(z_{1}(t), z_{2}(t)\right)$ be a solution of system (49) through $\left(l v_{1}, l v_{2}\right)$ for $t_{0}-\tau \leq t \leq t_{0}$, where $l>0$ satisfies $l v_{1}<y_{1}\left(t_{0}+\theta\right), l v_{2}<y_{2}\left(t_{0}+\theta\right)$ for $\theta \in[-\tau, 0]$. Since the semiflow of (49) is monotone and $A_{\varepsilon} v>0$, it follows from [14] that $z_{i}(t)$ is strictly increasing and $z_{i}(t) \rightarrow+\infty$ as $t \rightarrow$ $+\infty, i=1,2$. By comparison, $y_{1}(t) \rightarrow+\infty, y_{2}(t) \rightarrow+\infty$ as $t \rightarrow+\infty$, contradicting Lemma 8 . Hence, $W^{s}\left(\widehat{E}_{1}\right) \cap C^{0}=\phi$.

By Lemma 9, we conclude that $C_{0}$ repels positive solutions of system (3) uniformly. Hence, system (3) is permanent. This proof is complete.

\section{Global Stability}

In this section, we study the global stability of the predatorextinction equilibrium $E_{1}(r / a, 0,0)$ and the coexistence equilibrium $E^{*}=\left(x^{*}, y_{1}^{*}, y_{2}^{*}\right)$, respectively, by means of Lyapunov functionals and LaSalle's invariance principle.

First, we discuss the global stability of the predatorextinction equilibrium $E_{1}(r / a, 0,0)$.

Theorem 11. If $a_{2} r\left(r_{1}+D\right)<(a+m r)\left[r_{2}\left(r_{1}+D\right)-e D\right]$, then the predator-extinction equilibrium $E_{1}(r / a, 0,0)$ is globally asymptotically stable.

Proof. Let $\left(x(t), y_{1}(t), y_{2}(t)\right)$ be any positive solution of system (3) with initial conditions (4). By Theorem 1, we know that $E_{1}$ is locally asymptotically stable if $a_{2} r\left(r_{1}+D\right)<(a+$ $m r)\left[r_{2}\left(r_{1}+D\right)-e D\right]$.

Define

$$
V_{11}(t)=\frac{a_{2}}{1+m x_{0}}\left(x-x_{0}-x_{0} \ln \frac{x}{x_{0}}\right)+c y_{1}+a_{1} y_{2},
$$

where $x_{0}=r / a, c=a_{1} D /\left(D+r_{1}\right)$.

Calculating the derivative of $V_{11}(t)$ along positive solutions of system (3), we obtain that

$$
\begin{aligned}
& \frac{\mathrm{d}}{\mathrm{d} t} V_{11}(t) \\
& =\frac{a_{2}}{1+m x_{0}}\left(x(t)-x_{0}\right)\left[r-a x(t)-\frac{a_{1} y_{2}(t)}{1+m x}\right] \\
& \quad+c\left[e y_{2}(t)-\left(r_{1}+D\right) y_{1}(t)\right] \\
& \quad+a_{1}\left[D y_{1}(t)-r_{2} y_{2}(t)+\frac{a_{2} x(t-\tau) y_{2}(t-\tau)}{1+m x(t-\tau)}\right] .
\end{aligned}
$$

On substituting $r=a x_{0}$ into (52), we derive that

$$
\begin{aligned}
\frac{\mathrm{d}}{\mathrm{d} t} V_{11}(t)= & -\frac{a a_{2}}{1+m x_{0}}\left(x(t)-x_{0}\right)^{2} \\
& -\frac{a_{1} a_{2} y_{2}\left[x\left(1+m x_{0}\right)-x_{0}(1+m x)\right]}{(1+m x)\left(1+m x_{0}\right)} \\
& +c\left[e y_{2}(t)-\left(r_{1}+D\right) y_{1}(t)\right] \\
& +a_{1}\left[D y_{1}(t)-r_{2} y_{2}(t)+\frac{a_{2} x(t-\tau) y_{2}(t-\tau)}{1+m x(t-\tau)}\right] \\
= & -\frac{a a_{2}}{1+m x_{0}}\left(x(t)-x_{0}\right)^{2}-\frac{a_{1} a_{2} x y_{2}}{1+m x} \\
& +\left(\frac{a_{1} e D}{D+r_{1}}-a_{1} r_{2}+\frac{a_{1} a_{2} x_{0}}{1+m x_{0}}\right) y_{2} \\
& +\frac{a_{1} a_{2} x(t-\tau) y_{2}(t-\tau)}{1+m x(t-\tau)} .
\end{aligned}
$$

Define

$$
V_{1}(t)=V_{11}(t)+a_{1} a_{2} \int_{t-\tau}^{t} \frac{x(s) y_{2}(s)}{1+m x(s)} \mathrm{d} s .
$$


By (53) and (54), it follows that

$$
\begin{aligned}
& \frac{\mathrm{d}}{\mathrm{d} t} V_{1}(t) \\
& =-\frac{a a_{2}}{1+m x_{0}}\left(x(t)-x_{0}\right)^{2} \\
& \quad+\left(\frac{a_{1} e D}{D+r_{1}}-a_{1} r_{2}+\frac{a_{1} a_{2} x_{0}}{1+m x_{0}}\right) y_{2} \\
& =-\frac{a a_{2}}{1+m x_{0}}\left(x(t)-x_{0}\right)^{2} \\
& \quad+\frac{a_{1}\left[a_{2} r\left(D+r_{1}\right)-(a+m r)\left(r_{2}\left(r_{1}+D\right)-e D\right)\right]}{(a+m r)\left(r_{1}+D\right)} y_{2} .
\end{aligned}
$$

If $a_{2} r\left(r_{1}+D\right)<(a+m r)\left[r_{2}\left(r_{1}+D\right)-e D\right]$, then $\dot{V}_{1}(t) \leq$ 0 . By Theorem 5.3.1 in [11], solutions limit to $\mathscr{M}$, the largest invariant sunset of $\left\{\dot{V}_{1}(t)=0\right\}$. We can see from (55) that $\dot{V}_{1}(t)=0$ if and only if $x=x_{0}, y_{2}=0$. Since $\mathscr{M}$ is invariant, for each element in $\mathscr{M}$, we have $x=x_{0}, y_{2}=0$. Therefore, it follows from the last equation of system (3) that

$$
0=\dot{y}_{2}=D y_{1}(t) \text {, }
$$

which yields $y_{1}(t)=0$. Consequently, $\dot{V}_{1}(t)=0$ if and only if $x=x_{0}, y_{1}=0, y_{2}=0$. Hence, by Lasalle's invariance principle, $E_{1}(r / a, 0,0)$ is globally asymptotically stable. The proof is complete.

Next, we prove the global stability of the coexistence equilibrium $E^{*}=\left(x^{*}, y_{1}^{*}, y_{2}^{*}\right)$.

Theorem 12. If (H1) holds, then the coexistence equilibrium $E^{*}=\left(x^{*}, y_{1}^{*}, y_{2}^{*}\right)$ is globally asymptotically stable provided the following.

(H3) $\underline{x}>r /(2 a)$.

Here, $\underline{x}>0$ is the persistency constant for $x$ satisfying $\liminf \operatorname{int}_{t \rightarrow+\infty} x \geq \underline{x}$.

Proof. Let $\left(x(t), y_{1}(t), y_{2}(t)\right)$ be any positive solution of system (3) with initial conditions (4). Since $\underline{x}>r /(2 a)$, we know that there exists a $T_{4}>0$ such that $x(t)>r /(2 a)$ for $t>T_{4}$ and also that $x^{*}>r /(2 a)$. By Theorem 5 in [10], it is shown that $p_{2}+q_{2}>0,\left(p_{2}+q_{2}\right)\left(p_{1}+q_{1}\right)-\left(p_{0}+q_{0}\right)>0$. If (H1) holds, according to (21), $l_{0}>0, l_{1}>0, l_{2}>0$ for $x^{*}>r /(2 a)$. Then, (20) has no positive roots. Hence, $E^{*}$ is locally asymptotically stable for all $\tau \geq 0$.

Define

$$
\begin{aligned}
V_{21}(t)= & x-x^{*}-x^{*} \ln \frac{x}{x^{*}}+c_{1}\left(y_{1}-y_{1}^{*}-y_{1}^{*} \ln \frac{y_{1}}{y_{1}^{*}}\right) \\
& +c_{2}\left(y_{2}-y_{2}^{*}-y_{2}^{*} \ln \frac{y_{2}}{y_{2}^{*}}\right),
\end{aligned}
$$

where $c_{1}\left(D+r_{1}\right)=c_{2} D, c_{2}=a_{1}\left(1+m x^{*}\right) / a_{2}$.
Calculating the derivative of $V_{21}(t)$ along positive solutions of system (3), we obtain that

$$
\begin{aligned}
\frac{\mathrm{d}}{\mathrm{d} t} V_{21}(t) & \\
= & \left(1-\frac{x^{*}}{x}\right)\left[x(r-a x)-\frac{a_{1} x y_{2}}{1+m x}\right] \\
& +c_{1}\left(1-\frac{y_{1}^{*}}{y_{1}}\right)\left[e y_{2}-\left(r_{1}+D\right) y_{1}\right] \\
& +c_{2}\left(1-\frac{y_{2}^{*}}{y_{2}}\right)\left[D y_{1}-r_{2} y_{2}+\frac{a_{2} x(t-\tau) y_{2}(t-\tau)}{1+m x(t-\tau)}\right] .
\end{aligned}
$$

On substituting $x^{*}\left(r-a x^{*}\right)=a_{1} x^{*} y_{2}^{*} /\left(1+m x^{*}\right)$ into (58), we derive that

$$
\begin{aligned}
& \frac{\mathrm{d}}{\mathrm{d} t} V_{21}(t) \\
& =\left(1-\frac{x^{*}}{x}\right)\left[\left(x-x^{*}\right)\left(r-a\left(x+x^{*}\right)\right)+\frac{a_{1} x^{*} y_{2}^{*}}{1+m x^{*}}\right] \\
& -\frac{a_{1} x y_{2}}{1+m x}\left(1-\frac{x^{*}}{x}\right) \\
& +c_{1}\left[e y_{2}-e y_{1}^{*} \frac{y_{2}}{y_{1}}+\left(r_{1}+D\right) y_{1}^{*}\right] \\
& +c_{2}\left[-r_{2} y_{2}-D y_{2}^{*} \frac{y_{1}}{y_{2}}+r_{2} y_{2}^{*}+\frac{a_{2} x(t-\tau) y_{2}(t-\tau)}{1+m x(t-\tau)}\right. \\
& \left.-a_{2} y_{2}^{*} \frac{x(t-\tau) y_{2}(t-\tau)}{(1+m x(t-\tau)) y_{2}}\right] \\
& =\left(1-\frac{x^{*}}{x}\right)\left[\left(x-x^{*}\right)\left(r-a\left(x+x^{*}\right)\right)+\frac{a_{1} x^{*} y_{2}^{*}}{1+m x^{*}}\right] \\
& -\frac{a_{1} x y_{2}}{1+m x}\left(1+m x^{*}-\frac{x^{*}(1+m x)}{x}\right) \\
& +c_{1}\left[e y_{2}-e y_{1}^{*} \frac{y_{2}}{y_{1}}+\left(r_{1}+D\right) y_{1}^{*}\right] \\
& +c_{2}\left[-r_{2} y_{2}-D y_{2}^{*} \frac{y_{1}}{y_{2}}+r_{2} y_{2}^{*}\right. \\
& +\frac{a_{2} x(t-\tau) y_{2}(t-\tau)}{1+m x(t-\tau)} \\
& \left.-a_{2} y_{2}^{*} \frac{x(t-\tau) y_{2}(t-\tau)}{(1+m x(t-\tau)) y_{2}}\right] \\
& =\left(1-\frac{x^{*}}{x}\right)\left[\left(x-x^{*}\right)\left(r-a\left(x+x^{*}\right)\right)+\frac{a_{1} x^{*} y_{2}^{*}}{1+m x^{*}}\right] \\
& -\frac{a_{1} x y_{2}\left(1+m x^{*}\right)}{1+m x}+a_{1} x^{*} y_{2} \\
& +c_{1}\left[e y_{2}-e y_{1}^{*} \frac{y_{2}}{y_{1}}+\left(r_{1}+D\right) y_{1}^{*}\right]
\end{aligned}
$$




$$
\begin{aligned}
+c_{2}[ & -r_{2} y_{2}-D y_{2}^{*} \frac{y_{1}}{y_{2}}+r_{2} y_{2}^{*} \\
& +\frac{a_{2} x(t-\tau) y_{2}(t-\tau)}{1+m x(t-\tau)} \\
& \left.-a_{2} y_{2}^{*} \frac{x(t-\tau) y_{2}(t-\tau)}{(1+m x(t-\tau)) y_{2}}\right] .
\end{aligned}
$$

Noticing that $a_{1} x^{*}+e c_{1}=c_{2} r_{2}$, (59) can be rewritten as

$$
\begin{aligned}
\frac{\mathrm{d}}{\mathrm{d} t} V_{21}(t)= & \frac{\left(x-x^{*}\right)^{2}}{x}\left[r-a\left(x+x^{*}\right)\right] \\
& +\frac{a_{1} x^{*} y_{2}^{*}}{1+m x^{*}}\left(1-\frac{x^{*}}{x}\right)-\frac{a_{1}\left(1+m x^{*}\right)}{1+m x} x y_{2} \\
& +c_{1}\left[-e y_{2}^{*} \frac{y_{1}^{*}}{y_{2}^{*}} \frac{y_{2}}{y_{1}}+\left(r_{1}+D\right) y_{1}^{*}\right] \\
& +c_{2}\left[-D y_{1}^{*} \frac{y_{2}^{*}}{y_{1}^{*} \frac{y_{1}}{y_{2}}+r_{2} y_{2}^{*}}\right. \\
& +\frac{a_{2} x(t-\tau) y_{2}(t-\tau)}{1+m x(t-\tau)} \\
& \left.-a_{2} y_{2}^{*} \frac{x(t-\tau) y_{2}(t-\tau)}{(1+m x(t-\tau)) y_{2}}\right] .
\end{aligned}
$$

Define

$$
\begin{aligned}
V_{2}(t)=V_{21}(t) & \\
+a_{2} c_{2} \int_{t-\tau}^{t} & {\left[\frac{x(s) y_{2}(s)}{1+m x(s)}-\frac{x^{*} y_{2}^{*}}{1+m x^{*}}\right.} \\
& -\frac{x^{*} y_{2}^{*}}{1+m x^{*}} \\
& \left.\times \ln \frac{x(s) y_{2}(s)\left(1+m x^{*}\right)}{x^{*} y_{2}^{*}(1+m x(s))}\right] \mathrm{d} s .
\end{aligned}
$$

According to (60) and (61), it follows that

$$
\begin{aligned}
\frac{\mathrm{d}}{\mathrm{d} t} V_{2}(t)= & \frac{\left(x-x^{*}\right)^{2}}{x}\left[r-a\left(x+x^{*}\right)\right] \\
& +\frac{a_{1} x^{*} y_{2}^{*}}{1+m x^{*}}\left(1-\frac{x^{*}}{x}\right)-\frac{a_{1}\left(1+m x^{*}\right)}{1+m x} x y_{2} \\
& -c_{1} e y_{2}^{*} \frac{y_{1}^{*}}{y_{2}^{*}} \frac{y_{2}}{y_{1}}+c_{1}\left(r_{1}+D\right) y_{1}^{*}-c_{2} D y_{1}^{*} \frac{y_{2}^{*}}{y_{1}^{*}} \frac{y_{1}}{y_{2}} \\
& +c_{2} r_{2} y_{2}^{*}-a_{2} c_{2} y_{2}^{*} \frac{x(t-\tau) y_{2}(t-\tau)}{(1+m x(t-\tau)) y_{2}} \\
& +a_{2} c_{2} \frac{x y_{2}}{1+m x} \\
& -a_{2} c_{2} \frac{x^{*} y_{2}^{*}}{1+m x^{*}} \ln \frac{x(t) y_{2}(t)\left(1+m x^{*}\right)}{x^{*} y_{2}^{*}(1+m x(t))} \\
& +a_{2} c_{2} \frac{x^{*} y_{2}^{*}}{1+m x^{*}} \ln \frac{x(t-\tau) y_{2}(t-\tau)\left(1+m x^{*}\right)}{x^{*} y_{2}^{*}(1+m x(t-\tau))} .
\end{aligned}
$$

Noting that

$$
\begin{aligned}
c_{1} e y_{2}^{*}=c_{1}\left(r_{1}+D\right) y_{1}^{*} & =c_{2} D y_{1}^{*}, \quad c_{2} r_{2}=a_{1} x^{*}+e c_{1}, \\
a_{2} c_{2} & =a_{1}\left(1+m x^{*}\right), \\
\frac{a_{1} x^{*} y_{2}^{*}}{1+m x^{*}}\left(1-\frac{x^{*}}{x}\right) & =a_{1} x^{*} y_{2}^{*}\left(1-\frac{x^{*}(1+m x)}{x\left(1+m x^{*}\right)}\right),
\end{aligned}
$$

we derive from (62) that

$$
\begin{aligned}
\frac{\mathrm{d}}{\mathrm{d} t} V_{2}(t)= & \frac{\left(x-x^{*}\right)^{2}}{x}\left[r-a\left(x+x^{*}\right)\right] \\
& +c_{1} e y_{2}^{*}\left(2-\frac{y_{1}^{*}}{y_{2}^{*}} \frac{y_{2}}{y_{1}}-\frac{y_{2}^{*}}{y_{1}^{*}} \frac{y_{1}}{y_{2}}\right) \\
& -a_{1} x^{*} y_{2}^{*}\left[\frac{\left(1+m x^{*}\right) x(t-\tau) y_{2}(t-\tau)}{(1+m x(t-\tau)) x^{*} y_{2}}-1\right. \\
& \left.-\ln \frac{\left(1+m x^{*}\right) x(t-\tau) y_{2}(t-\tau)}{(1+m x(t-\tau)) x^{*} y_{2}}\right] \\
& -a_{1} x^{*} y_{2}^{*}\left[\frac{x^{*}(1+m x)}{x\left(1+m x^{*}\right)}-1-\ln \frac{x^{*}(1+m x)}{x\left(1+m x^{*}\right)}\right] .
\end{aligned}
$$

If $x(t)>r /(2 a)$ for $t>T_{4}$, then

$$
\frac{\left(x-x^{*}\right)^{2}}{x}\left[r-a\left(x+x^{*}\right)\right] \leq 0,
$$

and the equality holds only for $x=x^{*}$. Since the arithmetic mean is greater than or equal to the geometric mean, it is shown that

$$
2-\frac{y_{1}^{*}}{y_{2}^{*}} \frac{y_{2}}{y_{1}}-\frac{y_{2}^{*}}{y_{1}^{*}} \frac{y_{1}}{y_{2}} \leq 0,
$$

with equality if and only if $y_{1}^{*} y_{2} /\left(y_{2}^{*} y_{1}\right)=y_{2}^{*} y_{1} /\left(y_{1}^{*} y_{2}\right)$. Together with (64), it follows that if $x(t)>r /(2 a)$ for $t>T_{4}$, $\dot{V}_{2}(t) \leq 0$, with equality if and only if $x=x^{*}, y_{1}^{*} y_{2} /\left(y_{2}^{*} y_{1}\right)=$ $y_{2}^{*} y_{1} /\left(y_{1}^{*} y_{2}\right),\left(1+m x^{*}\right) x(t-\tau) y_{2}(t-\tau) /\left(x^{*} y_{2}(1+m x(t-\tau))\right)=$ 1. Hence, we now look for the invariant subset $\mathscr{M}$ within the set

$$
\begin{aligned}
N=\left\{\left(x, y_{1}, y_{2}\right): x=x^{*}, \frac{y_{1}^{*}}{y_{2}^{*}} \frac{y_{2}}{y_{1}}=\frac{y_{2}^{*}}{y_{1}^{*}} \frac{y_{1}}{y_{2}},\right. \\
\\
\left.\frac{\left(1+m x^{*}\right) x(t-\tau) y_{2}(t-\tau)}{(1+m x(t-\tau)) x^{*} y_{2}}=1\right\} .
\end{aligned}
$$

Since $x=x^{*}$ on $\mathscr{M}$, then $0=\dot{x}(t)=x^{*}\left(r-a x^{*}-a_{1} y_{2} /(1+\right.$ $\left.m x^{*}\right)$ ), which yields $y_{2}(t)=y_{2}^{*}$. It follows from the last equation of system (3) that $0=\dot{y}_{2}(t)=D y_{1}(t)-r y_{2}^{*}+$ $a_{2} x^{*} y_{2}^{*} /\left(1+m x^{*}\right)$, which yields $y_{1}(t)=y_{1}^{*}$. Thus, the only invariant set in $N$ is $\mathscr{M}=\left(x^{*}, y_{1}^{*}, y_{2}^{*}\right)$. Hence, by Lasalle's invariance principle, $E^{*}$ is globally asymptotically stable. The proof is complete. 
Remark 13. By Theorem 12, it is shown that if (H1) holds and $\underline{x}>r /(2 a)$, then the coexistence equilibrium $E^{*}=$ $\left(x^{*}, y_{1}^{*}, y_{2}^{*}\right)$ is globally asymptotically stable. We now give a sufficient condition for this inequality. By (35), $y_{2}(t)$ has an ultimately upper bound $M_{3}=a_{2}(r+\delta)^{2} /\left(4 a a_{1} \delta\right)$. Hence, we derive from the first equation of system (3) that $\dot{x}(t) \geq$ $x(t)\left(r-a x(t)-a_{1} M_{3}\right)$, which yields $\liminf _{t \rightarrow+\infty} x(t) \geq \underline{x}=$ $\left(r-a_{1} M_{3}\right) / a$. A brief calculation shows that we need only to choose $2 a r \delta>a_{2}(r+\delta)^{2}$; then $\underline{x}>r /(2 a)$.

\section{Acknowledgments}

This work was supported by the National Natural Science Foundation of China (11071254) and the Nature Science Foundation for Yong Scientists of Hebei Province, China (A2013506012).

\section{References}

[1] S. Zhang, D. Tan, and L. Chen, "Chaos in periodically forced Holling type II predator-prey system with impulsive perturbations," Chaos, Solitons and Fractals, vol. 28, no. 2, pp. 367-376, 2006.

[2] C. S. Holling, "The functional response of predator to prey density and its role in mimicry and population regulation," Memoirs of the Entomological Society of Canada, vol. 45, pp. 160, 1965.

[3] R. Xu, M. A. J. Chaplain, and F. A. Davidson, "Periodic solutions for a predator-prey model with Holling-type functional response and time delays," Applied Mathematics and Computation, vol. 161, no. 2, pp. 637-654, 2005.

[4] W. Ko and K. Ryu, "Qualitative analysis of a predator-prey model with Holling type II functional response incorporating a prey refuge," Journal of Differential Equations, vol. 231, no. 2, pp. 534-550, 2006.

[5] K. Gopalsamy, Stability and Oscillations in Delay Differential Equations of Population Dynamics, Kluwer Academic, Dordrecht, The Netherlands, 1992.

[6] Y. Kuang, Delay Differential Equations with Applications in Population Dynamics, Academic Press, Boston, Mass, USA, 1993.

[7] P. J. Wangersky and W. J. Cunningham, "Time lag in preypredator population models," Ecology, vol. 38, pp. 136-139, 1957.

[8] W. Wang, "Global dynamics of a population model with stage structure for predator," in Advanced Topics in Biomathematics: Proceeding of the International Conference on Mathematical Biology, L. Chen, S. Ruan, and J. Zhu, Eds., pp. 253-257, World Scientific, Singapore, 1997.

[9] P. Georgescu and Y.-H. Hsieh, "Global dynamics of a predatorprey model with stage structure for the predator," SIAM Journal on Applied Mathematics, vol. 67, no. 5, pp. 1379-1395, 2007.

[10] X. Tian and R. Xu, "Global dynamics of a predator-prey system with Holling type II functional response," Nonlinear Analysis: Modelling and Control, vol. 16, no. 2, pp. 242-253, 2011.

[11] J. K. Hale, Theory of Functional Differential Equations, Springer, New York, NY, USA, 1976.

[12] S. Ruan and J. Wei, "On the zeros of a third degree exponential polynomial with applications to a delayed model for the control of testosterone secretion," IMA Journal of Mathemathics Applied in Medicine and Biology, vol. 18, no. 1, pp. 41-52, 2001.
[13] J. K. Hale and P. Waltman, "Persistence in infinite-dimensional systems," SIAM Journal on Mathematical Analysis, vol. 20, no. 2, pp. 388-395, 1989.

[14] H. L. Smith, "Monotone semiflows generated by functionaldifferential equations," Journal of Differential Equations, vol. 66, no. 3, pp. 420-442, 1987. 


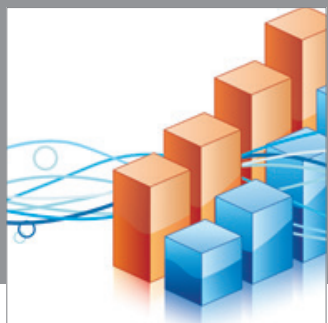

Advances in

Operations Research

mansans

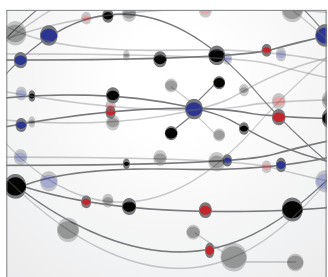

The Scientific World Journal
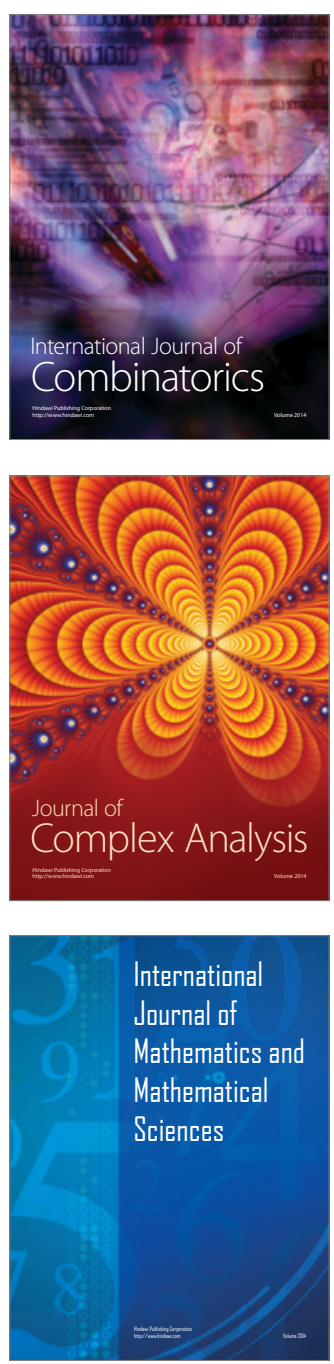
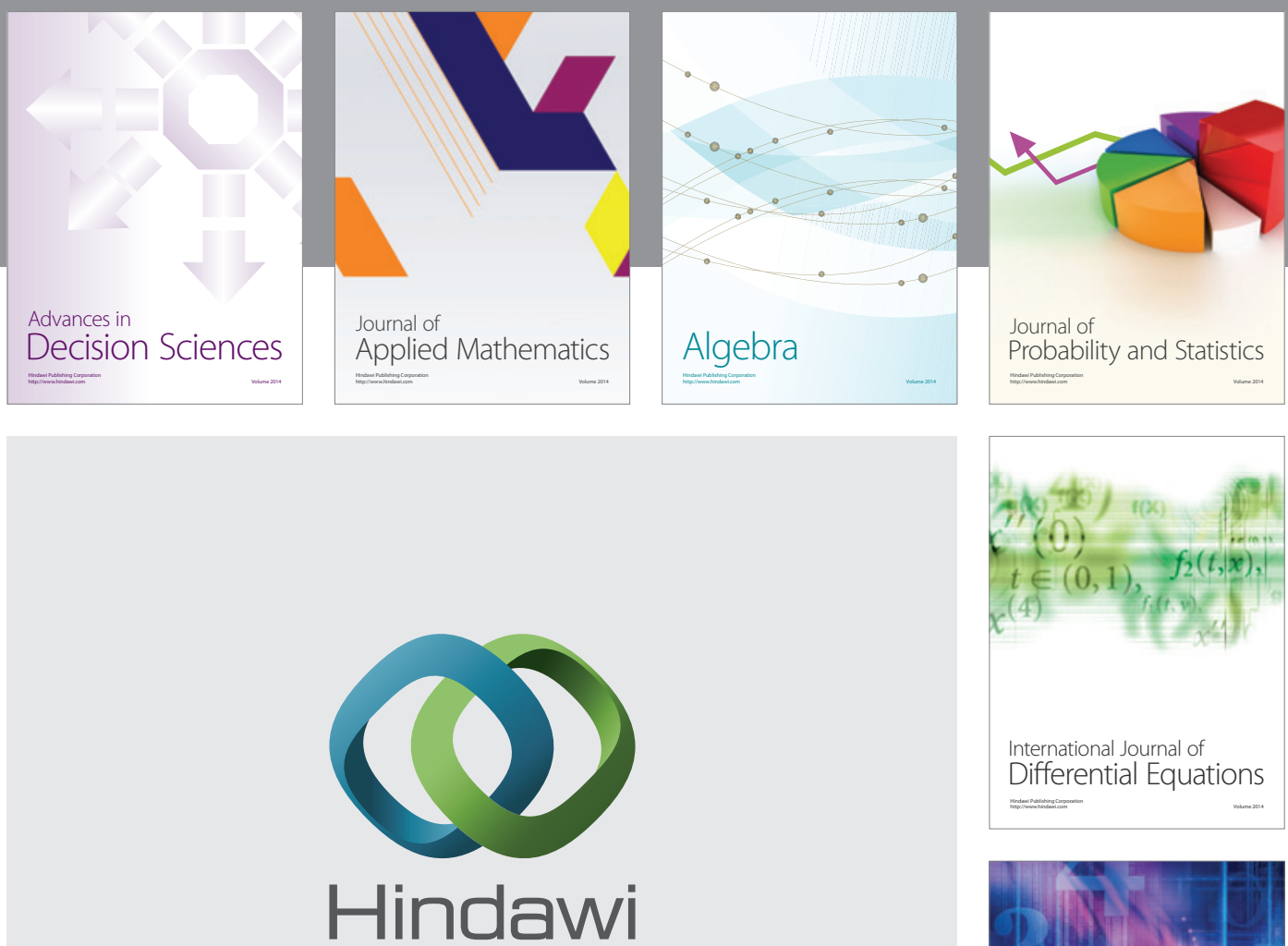

Submit your manuscripts at http://www.hindawi.com
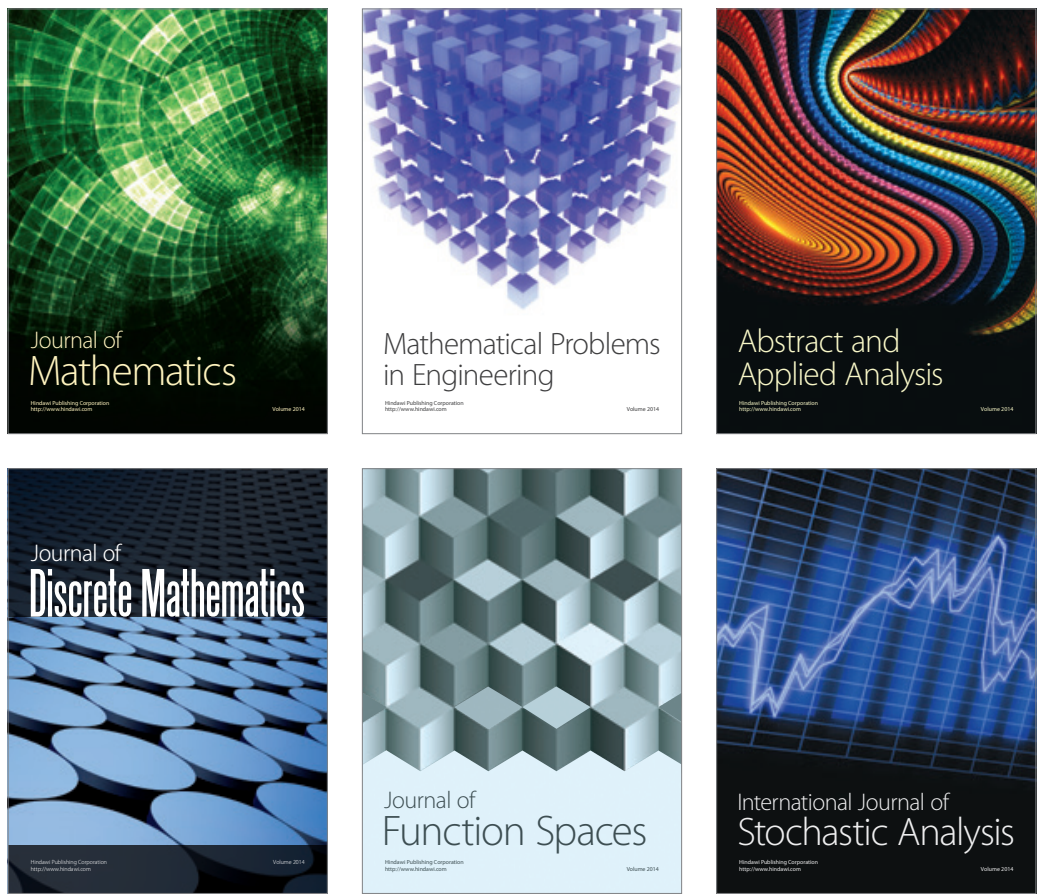

Journal of

Function Spaces

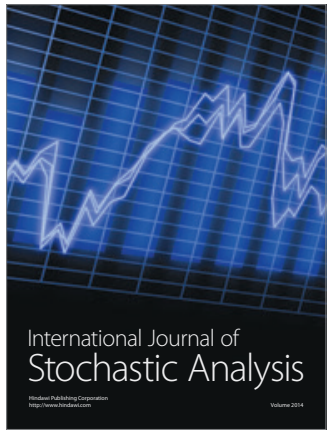

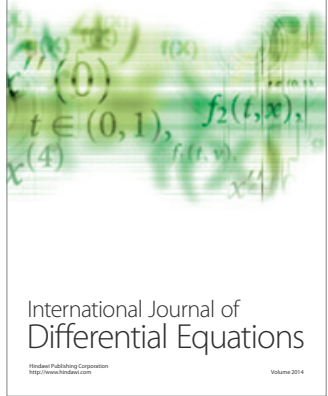
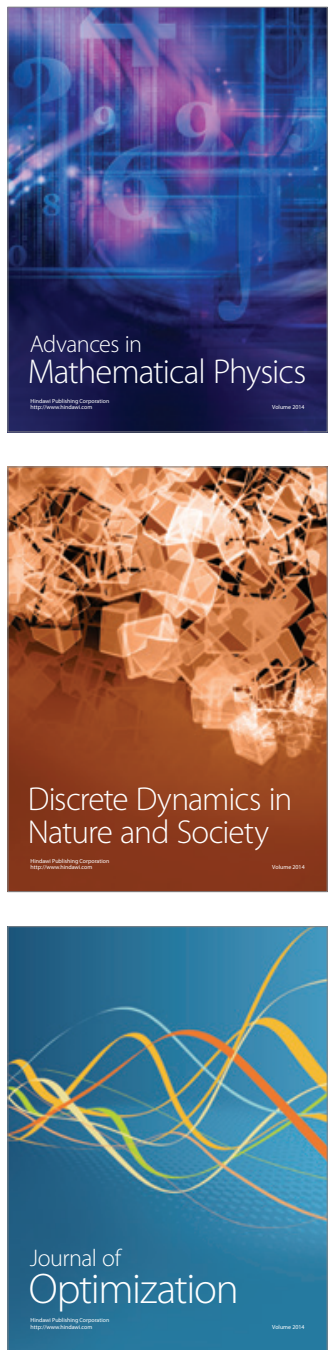